\title{
Transformation From Adenocarcinoma to Pleomorphic Carcinoma as an Acquired Resistance to Epidermal Growth Factor Receptor Tyrosine Kinase Inhibitors
}

\author{
Kanako Nishimatsu ${ }^{\mathrm{a}}$, Seigo Minami ${ }^{\mathrm{a}, \mathrm{c}}$, Shoichi Ihara ${ }^{\mathrm{a}}$, Hironao Yasuoka ${ }^{\mathrm{b}}$
}

\begin{abstract}
Pulmonary pleomorphic carcinoma is a very rare histological type of primary lung cancer, and usually provides aggressive clinical courses. A 65-year-old Japanese woman was diagnosed by transbronchial biopsy of the primary tumor as c-stage IV (cT4N3M1b) of adenocarcinoma harboring L858R point mutation in the exon 21 of epidermal growth factor receptor (EGFR). She received EGFR tyrosine kinase inhibitors (TKIs) (gefitinib and erlotinib) and subsequently cytotoxic chemotherapies. Gefitinib achieved partial response, but was switched to erlotinib due to elevated serum aspartate transaminase. After resistance to EGFR-TKIs, the second transbronchial re-biopsy revealed pulmonary pleomorphic carcinoma. Both carcinomatous and sarcomatous components retained the L858R mutation, but did not acquire T790M mutation. This case suggested that the histological transformation to pulmonary pleomorphic carcinoma may be one of mechanisms of drug resistance to EGFR-TKIs.
\end{abstract}

Keywords: Epidermal growth factor receptor mutation; Tyrosine kinase inhibitor; L858R point mutation; Pulmonary pleomorphic carcinoma; Adenocarcinoma; Drug resistance; Histological transformation; Gefitinib; Erlotinib; Transbronchial biopsy

\section{Introduction}

Epidermal growth factor receptor tyrosine kinase inhibitors (EGFR-TKIs) are indispensable drugs for patients with nonsmall cell lung cancer harboring active EGFR mutations. These drugs are superior in efficacy and survival benefit to conventional cytotoxic chemotherapy. However, the tumor becomes resistant to TKIs someday, and then progresses ap-

Manuscript submitted April 30, 2021, accepted June 3, 2021

Published online July 3, 2021

aDepartment of Respiratory Medicine, Osaka Police Hospital, Osaka, Japan ${ }^{b}$ Department of Pathology, Osaka Police Hospital, Osaka, Japan

${ }^{\mathrm{c} C}$ Corresponding Author: Seigo Minami, Department of Respiratory Medicine, Osaka Police Hospital, 10-31 Kitayama-cho, Tennoji-ku, Osaka-City, Osaka 543-0035, Japan. Email: seigominami@oph.gr.jp

doi: https://doi.org/10.14740/jmc3710 proximately 1 - 2 years after introduction of TKIs. Major acquired resistance mechanisms to EGFR-TKIs include T790M point mutation [1], overexpressed signaling of c-MET [2] and hepatocyte growth factor receptor [3]. Transformation to other aggressive histological types such as small cell carcinoma is also one of minor resistant mechanisms [4].

Pulmonary pleomorphic carcinoma histologically consists of carcinomatous tumor and at least $10 \%$ of sarcomatoid tumor components [5]. This tumor accounts for only $0.1-1.6 \%$ of all malignant lung tumors. It is highly resistant to chemotherapy and has unfavorable prognosis. However, it remains unknown whether EGFR-TKIs are effective against pleomorophic carcinoma harboring active EGFR mutation.

We herein report a case of possible resistance mechanism to EGFR-TKI by histopathological transformation from adenocarcinoma to pleomorphic carcinoma.

\section{Case Report}

\section{Investigations}

A 65-year-old woman, a current smoker, visited our hospital because of persistent cough, back pain and enlarged right supraclavicular lymph node. The chest computed tomography detected a tumor in the left hilar portion (Fig. 1a).

\section{Diagnosis}

She was diagnosed of adenocarcinoma (c-stage IV, cT4N3M1b in the seventh edition of UICC-TNM classification) by trans-bronchial biopsy. The tumor was bronchoscopically visible at the left main bronchus. A L858R point mutation in the exon 21 was detected by the peptide nucleic acid-locked nucleic acid polymerase chain reaction clamp method (by LSI Medience Corporation, Tokyo). The histological specimen showed diffuse staining with mutation-specific antibody for L858R (clone 43B2 rabbit monoclonal antibody, Cell Signaling Technology, Massachusetts, USA) (Fig. 2). Thereafter, gefitinib achieved partial response (Fig. 1b).

Twenty months after the initiation of gefitinib, when the tumor was already resistant to EGFR-TKIs and cytotoxic chemotherapies, we performed re-biopsy from the intra-bron- 
chial primary lung tumor in the left main bronchus. The tumor specimen showed pleomorphic carcinoma consisting of adenocarcinomatous component and sarcomatous component. Both components were positively stained with specific antibody for L858R point mutation (Fig. 3). We did not detect T790M point mutation in the specimen by Cobas EGFR Mutation Test version 2 (by LSI Medience Corporation).

\section{Treatment}

She started gefitinib in October 2014 and achieved partial response (Fig. 1b). However, we switched gefitinib to erlotinib 3 months after the initiation of gefitinib, because serum aspartate transaminase elevated up to $204 \mathrm{U} / \mathrm{L}$. The primary tumor enlarged and we stopped erlotinib 9 months after the start of gefitinib. Thereafter, she received four cycles of combination chemotherapy of cisplatin and pemetrexed and subsequently three cycles of maintenance pemetrexed monotherapy. This cytotoxic regimen provided stable disease, but we found progressive disease in the primary tumor in the left lower lobe. Subsequent regimen of docetaxel monotherapy was discontinued because of enlarged primary tumor after the second cycles (Fig. 1c). At this time, we performed the second re-biopsy by bronchoscopy.

\section{Follow-up and outcome}

Afatinib did not respond to the transformed tumor and resulted in progressive disease after 3 months of afatinib treatment. Thereafter, she received best supportive care alone, and died of cancer progression 23 months after the initiation of gefitinib.

\section{Discussion}

This case was interesting in that the pretreatment pathological diagnosis was adenocarcinoma, but post-treatment histology was pleomorphic carcinoma after progression to EGFR-TKIs. There were many case reports of pleomorphic carcinoma harboring active EGFR mutations at the diagnosis. However, to our knowledge, there were only three previous case reports in which, during the resistance process to EGFR-TKIs, the tumor had transformed histologically from adenocarcinoma to pleomorophic carcinoma [6-8] (Table 1).

Our case suggested that transformation to more aggressive histological type, pleomorphic carcinoma, is a possible mechanism of drug resistance to EGFR-TKI. In our case, before and after EGFR-TKI and series of cytotoxic chemotherapy regimens, we bronchoscopically obtained tumor specimens from the same primary tumor. The histological comparison of these two specimens showed a change from adenocarcinoma to pleomorphic carcinoma. In the pleomorophic carcinoma obtained after EGFR-TKI, both carcinomatous and sarcomatous components retained EGFR L858R point mutation, but did not acquire T790M mutation.

A mechanism of histological transformation from adenocarcinoma to pleomorphic carcinoma may be various among
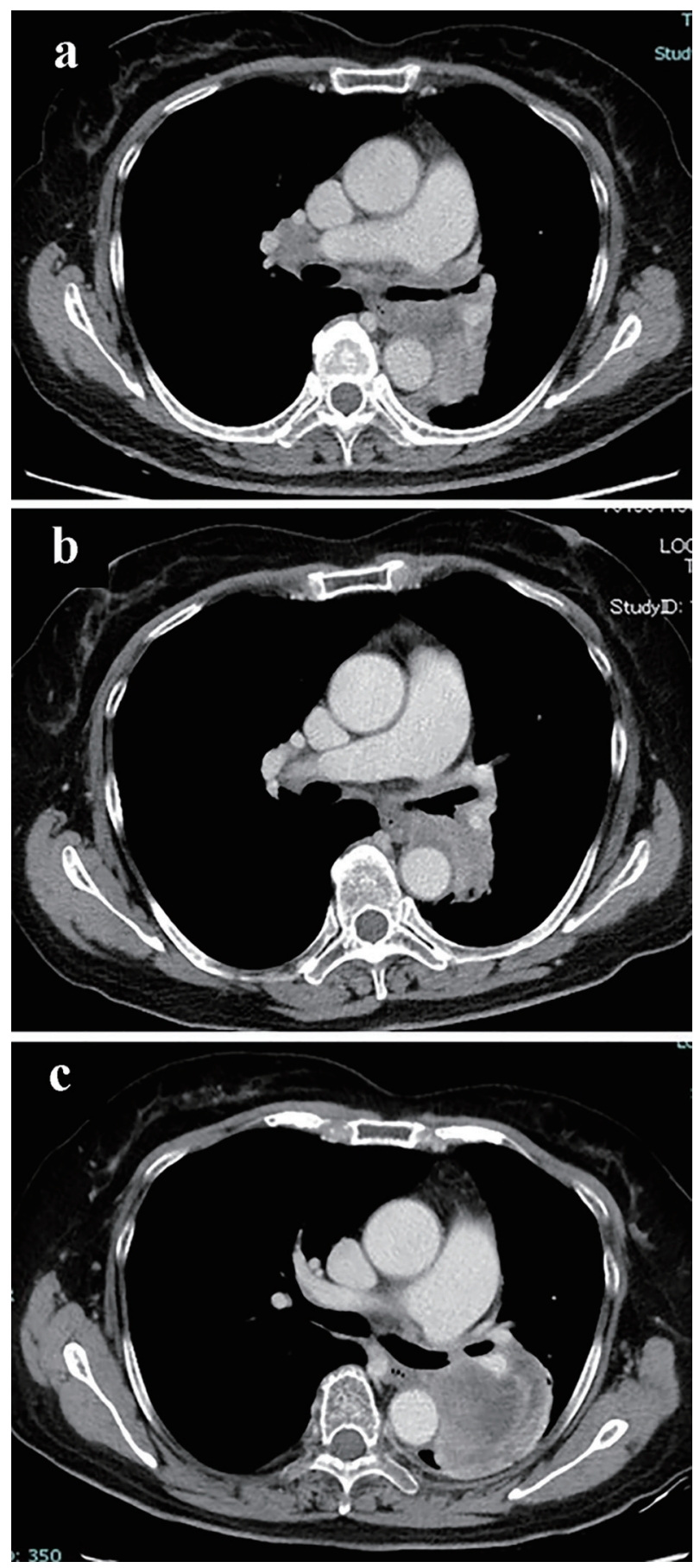

Figure 1. Chest CT scan (a) at the diagnosis, (b) 3 months after the initiation of gefitinib, and (c) at the second re-biopsy after resistance to EGFR-TKIs. CT: computed tomography; EGFR-TKI: epidermal growth factor receptor tyrosine kinase inhibitor.

cases. In a 65-year-old Japanese woman, transbronchial biopsy at diagnosis detected some spindle cells in the primarily adenocarcinomatous cells. Her autopsy specimens showed that the mutated EGFR immunohistochemically expressed only in the adenocarcinomatous component, but not in the spindle/sarcomatous components [7]. In this case, heterogeneity 

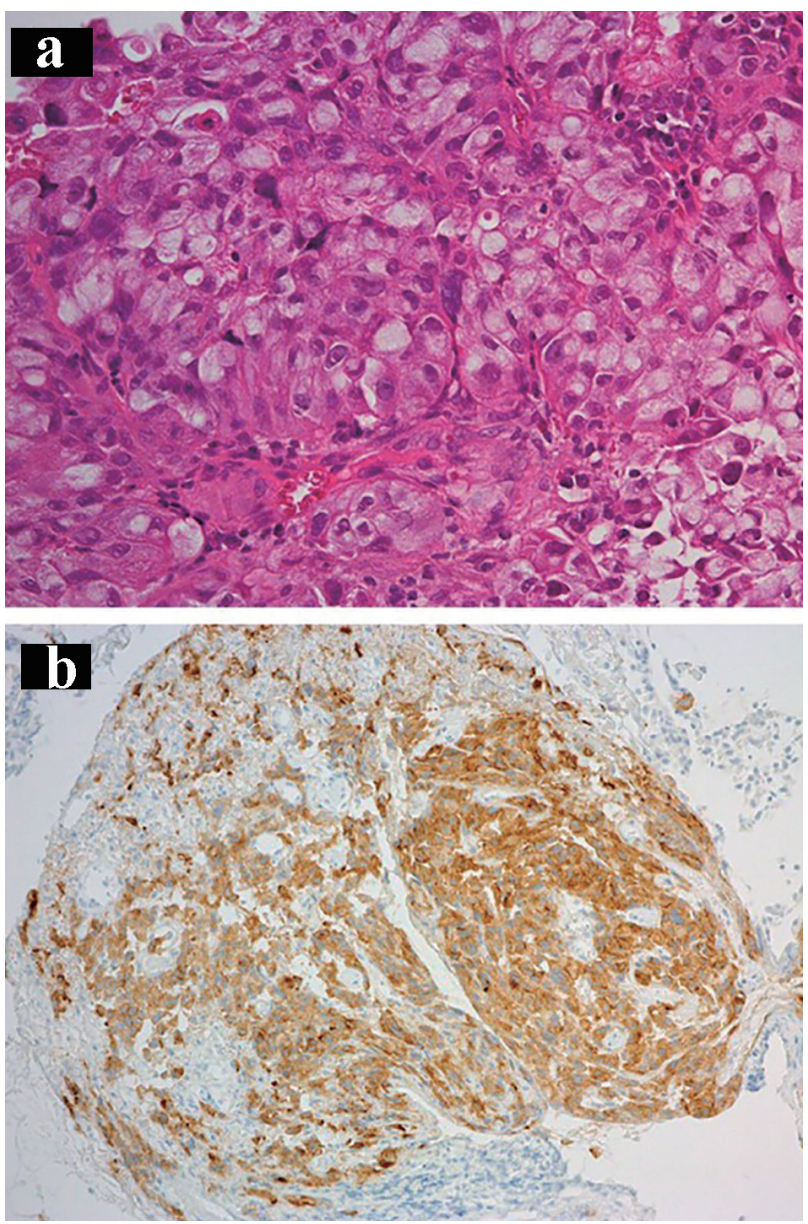

Figure 2. The first transbronchial biopsy specimen at the diagnosis shows adenocarcinoma: (a) hematoxylin and eosin staining $(\times 20)$ and (b) staining by anti-EGFR exon 21 specific antibody ( $\times 10)$. EGFR: epidermal growth factor receptor

of a main component of adenocarcinoma and a minor component of sarcomatous tumor in the original and primary tumor might be associated with resistance to gefitinib. In this speculation, sarcomatous tumor progressed against gefitinib, while adenocarcinoma component reduced by gefitinib. Thus, a main component might change from adenocarcinoma to pleomorphic carcinoma during gefitinib treatment. Another case of a 58-year-old Japanese man showed genetic heterogeneity of EGFR mutation status in the postmortem specimen. The sarcomatoid spindle cell component had both an exon 19 deletion and T790M point mutation of EGFR, while the adenocarcinomatous tumor component had only the exon 19 deletion [6]. The other 72-year-old Japanese woman was diagnosed as adenocarcinoma in the primary lung tumor, but then encountered pleomorphic sarcoma in the femoral metastasis. Gefitinib managed to reduce her primary lung tumor and other metastatic lesions, but failed to control the femoral metastasis. The same types of mutations in EGFR (L858R) and TP53 (R181P) were genetically detected in both the lung and metastatic tumors [8]. The latter two cases were similar to our case in that mutated EGFR expressed both in adenomatous and
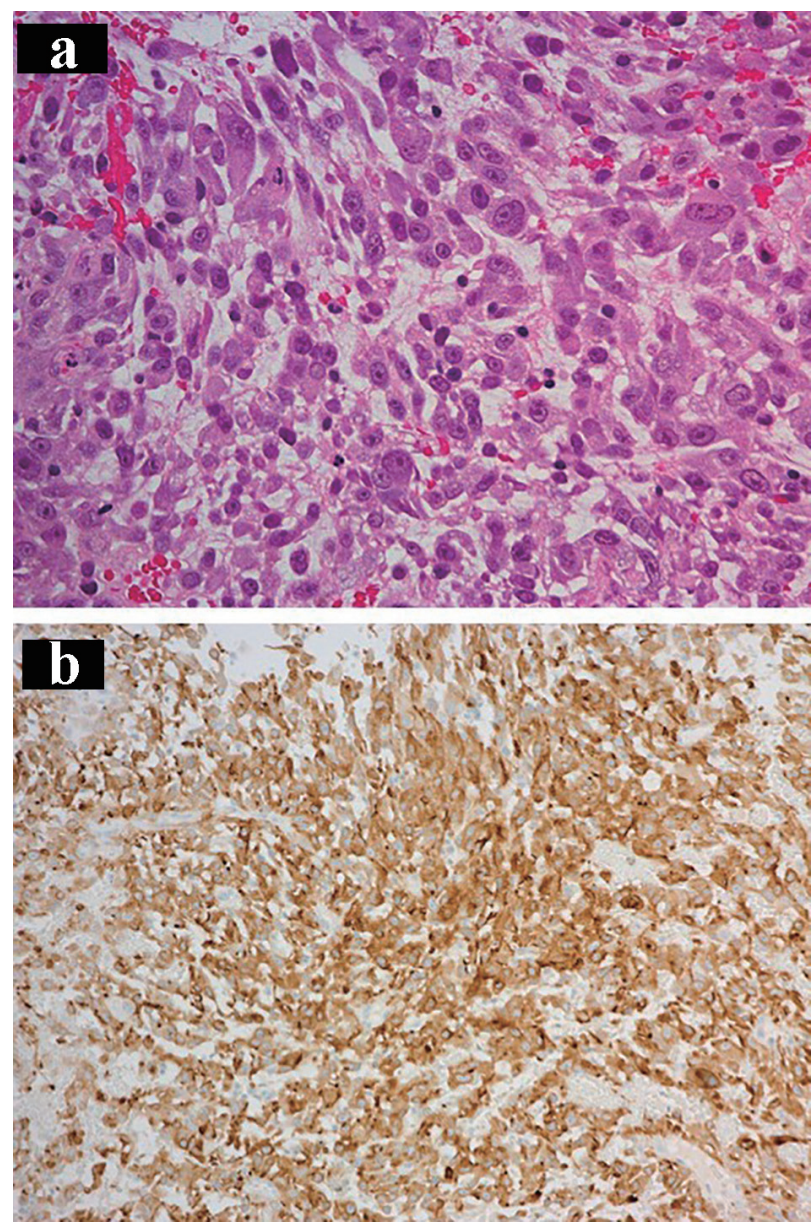

Figure 3. The second transbronchial re-biopsy specimen after resistance to EGFR-TKIs: (a) hematoxylin and eosin staining $(\times 20)$ and $(b)$ staining by anti-EGFR exon 21 specific antibody $(\times 10)$. EGFR-TKI: epidermal growth factor receptor tyrosine kinase inhibitor.

sarcomatous components. In a 60-year-old Japanese woman with pulmonary pleomorphic carcinoma, EGFR L858R point mutation expressed both in separately microdissected specimens from adenocarcinoma and spindle cell components [9]. According to the study by Chang, identical mutation of EGFR and p53 was observed both in carcinomatous and sarcomatous components in three of 42 patients with pleomorphic carcinomas [10]. These findings suggested monoclonal histogenesis, which means that sarcomatous component is originated from carcinomatous component.

Our case included the following two limitations. 1) We did not evaluate next-generation sequencing and other possible resistant mechanisms to EGFR-TKIs, such as c-MET amplification, hepatocyte growth factor over-expression and $K$-ras mutation, because of time-related deterioration (more than 5 - 6.5 years after the first and second biopsy) and insufficient volume of the remaining biopsy specimens. 2) Our pathological diagnoses were based on tiny specimens obtained by transbronchial biopsy. Precise diagnosis of pleomorphic carcinoma requires larger specimen, but it is usually difficult to obtain it in advanced lung cancer. 
Table 1. Summary of Previous Cases of Histological Changes From Adenocarcinoma to Pleomorphic Carcinoma After EGFR-TKIs

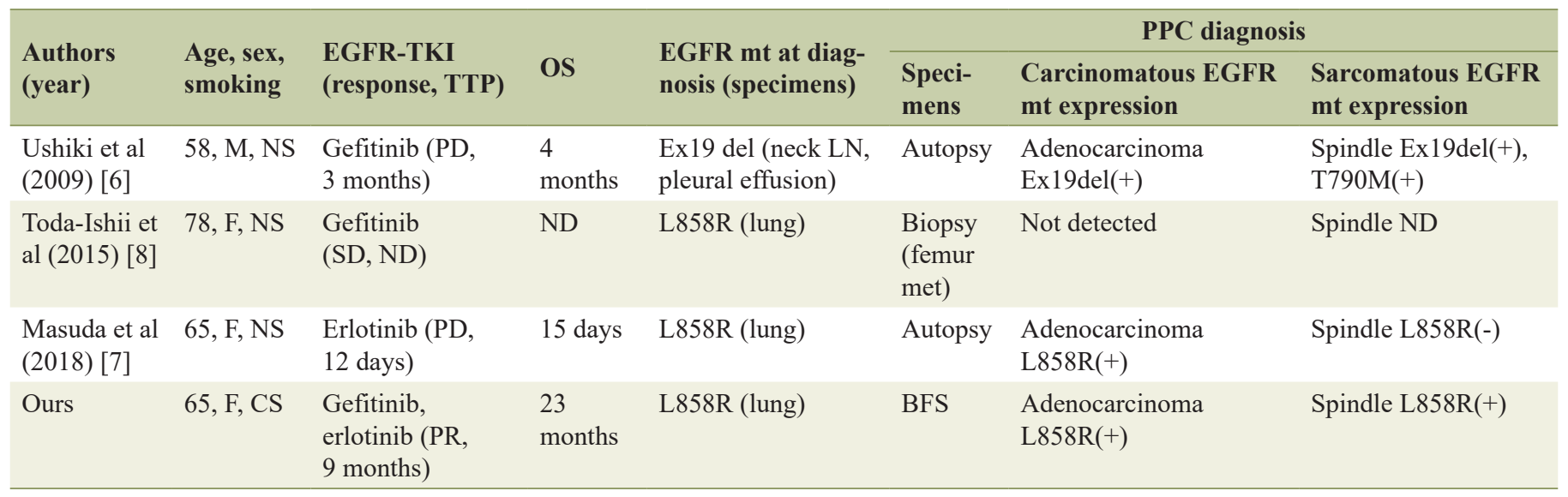

Adeno: adenocarcinoma; BFS: bronchoscopy; CS: current smoker; EGFR-TKI: epidermal growth factor receptor tyrosine kinase inhibitor; F: female; LN: Iymph node; M: male; met: metastasis; mt: mutation; ND: not described; NS: non-smoker; OS: overall survival; PD: progressive disease; PPC: pulmonary pleomorphic carcinoma; PR: partial response; SD: stable disease; TTP: time to progression.

\section{Conclusions}

This case suggested that the transformation to pulmonary pleomorphic carcinoma can be one of mechanisms of drug resistance to EGFR-TKIs.

\section{Acknowledgments}

We thank Dr. Kiyoshi Komuta (Department of Respiratory Medicine, Osaka Police Hospital at that time, and Osaka AntiTuberculosis Association Osaka Hospital at present) for clinically helpful advice, and Masahiko Tsujimoto (Department of Pathology, Osaka Police Hospital at that time) for his pathological diagnosis.

\section{Financial Disclosure}

None to declare.

\section{Conflict of Interest}

None to declare.

\section{Informed Consent}

Not applicable because the manuscript has been sufficiently de-identified to protect the patient.

\section{Author Contributions}

NK, IS and MS were mainly involved in diagnosis and treatment of this patient. MS and NK drafted the report. All authors read and critically reviewed the manuscript, and then approved the final submitted version.

\section{Data Availability}

The authors declare that data supporting the findings of this study are available within the article.

\section{References}

1. Kobayashi S, Boggon TJ, Dayaram T, Janne PA, Kocher $\mathrm{O}$, Meyerson M, Johnson BE, et al. EGFR mutation and resistance of non-small-cell lung cancer to gefitinib. N Engl J Med. 2005;352(8):786-792.

2. Engelman JA, Zejnullahu K, Mitsudomi T, Song Y, Hyland C, Park JO, Lindeman N, et al. MET amplification leads to gefitinib resistance in lung cancer by activating ERBB3 signaling. Science. 2007;316(5827):1039-1043.

3. Yano S, Wang W, Li Q, Matsumoto K, Sakurama H, Nakamura T, Ogino H, et al. Hepatocyte growth factor induces gefitinib resistance of lung adenocarcinoma with epidermal growth factor receptor-activating mutations. Cancer Res. 2008;68(22):9479-9487.

4. Sequist LV, Waltman BA, Dias-Santagata D, Digumarthy $\mathrm{S}$, Turke AB, Fidias P, Bergethon K, et al. Genotypic and histological evolution of lung cancers acquiring resistance to EGFR inhibitors. Sci Transl Med. 2011;3(75):75ra26.

5. Travis WD, Brambilla E, Nicholson AG, Yatabe Y, Austin JHM, Beasley MB, Chirieac LR, et al. The 2015 World Health Organization Classification of lung tumors: impact of genetic, clinical and radiologic advances since the 2004 classification. J Thorac Oncol. 2015;10(9):12431260.

6. Ushiki A, Koizumi T, Kobayashi N, Kanda S, Yasuo M, Yamamoto H, Kubo K, et al. Genetic heterogeneity of EGFR mutation in pleomorphic carcinoma of the lung: 
response to gefitinib and clinical outcome. Jpn J Clin Oncol. 2009;39(4):267-270.

7. Masuda K, Tokito T, Azuma K, Yanagida E, Nakamura M, Naito Y, Matsuo N, et al. Pulmonary pleomorphic carcinoma: A case harboring EGFR mutation treated with EGFR-TKIs. Thorac Cancer. 2018;9(6):754-757.

8. Toda-Ishii M, Akaike K, Kurisaki-Arakawa A, Arakawa A, Mukaihara K, Suehara Y, Takagi T, et al. Sarcomatous transformation of EGFR and TP53 mutation-positive metastatic adenocarcinoma of the lungs, masquerading as a primary pleomorphic sarcoma of the proximal femur. Int J Clin Exp Pathol. 2015;8(3):3270-3278.

9. Saitoh M, Niijima M, Takiguchi Y, Hiroshima K, Fujita Y, Nishio K, Tatsumi K. An early event of EGFR mutation in pleomorphic carcinoma of the lung. Int J Clin Oncol. 2011;16(6):770-773.

10. Chang YL, Wu CT, Shih JY, Lee YC. EGFR and p53 status of pulmonary pleomorphic carcinoma: implications for EGFR tyrosine kinase inhibitors therapy of an aggressive lung malignancy. Ann Surg Oncol. 2011;18(10):2952-2960. 